\title{
Assessment of recovery from serious head injury
}

\author{
MORRIS E. ESON, JOHN K. YEN, AND ROBERT S. BOURKE \\ From the State University of New York at Albany, and Division of Neurosurgery, \\ Albany Medical College, Albany, New York, USA
}

SUMMARY A procedure for serial assessment of neuropsychological recovery after serious head injury was designed. The assessment procedure consists of four segments, each appropriate for different phases of the recovery process. Recovery can be traced from early in the period of post-traumatic amnesia until it reaches an asymptote. The course of recovery of several patients has been observed. The procedure is shown to be practical and appears to be valid. The recovery process is compared to ontogenesis, and is shown to be generally similar though differing in important particulars.

Improved life support technology and patient management have resulted in the survival of ever increasing numbers of seriously head-injured patients (Bond and Brooks, 1976; Jennett et al., 1976). The quality of neuropsychological recovery in patients is highly variable, and the factors accounting for the differences in rate, pattern, and ultimate level of recovery are largely unknown. Systematic study of the process of recovery may provide a basis for more effective treatment regimes.

The usual neuropsychological assessment procedures are of limited applicability for the evaluation of the recently head-injured patient. The widely used battery of tests developed by Halstead and Reitan (Reitan and Davison, 1974), and its modification by Russell et al. (1970), are designed for administration to alert patients with relatively fixed focal deficits. The Halstead Impairment Index is useful for gross diagnostic determinations of locus and extent of brain lesions. Luria's procedure (Christenson, 1975; Luria and Majovski, 1977), although broader in its scope, is also aimed at a determination of the behavioural consequences of a static deficit. These assessment procedures are not designed to study the evolving improvement of function in the patient with altered levels of alertness and attention span. Moreover, the administration of these test batteries takes several hours, making them impractical for serial testing to

Address for reprint requests: Dr Morris E. Eson, Department of Psychology, State University of New York at Albany, 1400Washington Avenue, Albany, New York 12222, USA.

Accepted 27 June.1978 evaluate time-related improvement. Most patients with acceleration/deceleration head injuries suffer $\stackrel{\oplus}{\longrightarrow}$ focal, scattered histopathological lesions with $\overrightarrow{0}$ generalised neurological dysfunction rather than focal single anatomical lesions with solitary neurological deficits (Graham and Adams, 1971).

Neuropsychological studies of severely headinjured patients that have employed longitudinal or serial assessments have measured changes inspecific functions, such as memory (Brooks, 1974a, b, 1975, 1976; Levin et al., 1976; Hannayo극 et al., 1977) or selective attention and choice reaction time (Gronwall and Sampson, 1974). Studies of the recovery of intellectual function in general are remarkably few. One of these earlier studies (Ruesch and Moore, 1943) demonstrated that the duration of coma after injury was predictive of the rate and quality of recovery of intellectual function. Recently Mandleberg and Brooks (1975) traced the natural course of recovery of intellectual function after severe head injury by serial administration of the Wechsler Adult Intelligence Scale. They found that functions tested by the verbal subtests demonstrated less initial impairment and faster recovery than those functions tested by the non-verbal subtests. In addition, Mandleberg (1975) compared Wechsler Scale test scores of head-injured patients exhibiting clinical signs of post-traumatic amnesia with those who had recovered beyond that point. He concluded that the period of post-traumatic amnesia was a qualitatively distinct phase of recovery in head injury. Although these kinds of assessments demonstrate a pattern in recovery from severe 
head injury, they do not focus on generalised assessment of adaptive function.

The level of adaptive function varies with the stages of recovery from serious head injury. In the early stages, it is manifested in the ability to carry on the activities of daily living, self-care, and rudimentary social interaction. In the middle stages recovery of adaptive function is best measured indirectly by assessment of conceptual and information processing skills that underlie more mature social interaction and ability to initiate and carry out sustained planned activities directed toward a goal. Complete or nearly complete recovery of adaptive function is shown when the individual is able to seek and maintain employment, and is able to participate in normal adult social and recreational activities without supervision. This latter level we refer to as basic adaptive function. The relationship of the performance on a single intelligence scale such as the Wechsler and the level of adaptive function at various stages of recovery in the recently head-injured has not been established. Furthermore, any particular test of cognitive function, such as the Wechsler Adult Scale, is designed for restricted ranges of cognitive function in the alert subject and is not suitable for assessment of levels of function that deviate markedly from these ranges, particularly in patients with altered levels of consciousness and restricted attention span. In attempting to understand the various phases of cognitive and adaptive function throughout the course of recovery it would be desirable to use tests designed specifically for the assessment of the patient during each phase of neuropsychological recovery (Luria and Majovski, 1977). Those studies that have been concerned with the recovery of adaptive function after serious head injury (Bond, 1975; Bond and Brooks, 1976; Najenson et al., 1973, 1974, 1975) were aimed at the evaluation of patient status in terms of social and vocational adjustment six months to five years after injury. The process of recovery of adaptive function from shortly after injury was not investigated.

The scale we have designed is modelled on a developmental scale, with test instruments appropriate for each level of the recovery process. The first segment of the scale assesses the recovery during the period of post-traumatic amnesia, a period for which no assessment procedures are currently available. The four segments of the scale are linked to each other as measures of the recovery of adaptive function paralleling that of ontogenesis. Recovery since time of injury is plotted against months equivalent in development from zero to 200 months. The scale has general applicability as long as the premorbid social history reveals at least functional literacy, determined by educational and occupational data. Since the assessment procedure concerns itself with the recovery of basic adaptive functions, it becomes unnecessary to ascertain the extent to which the particular patient's premorbid function may have surpassed this basic level.

\section{Methods}

The scale is designed to be used from the time the patient recovers from the coma and sequentially to the time at which full recovery is demonstrated (basic adaptive function present in premorbid state) or until the patient's function fails to show improvement over a period of two to three months. The items are arranged according to a developmental sequence such that the demonstration of the presence of a more advanced function can be taken as evidence for the presence of a less advanced one. The early segments of this scale allow for the use of the impressions of the nursing staff looking after the patient. The scale is constructed in such a way that observation or test items are seldom repeated as long as the patient shows improvement, thus avoiding practice effects, and allowing for the completion of each evaluation session in 40 minutes or less (with the exception of the last segment which takes about one hour).

The scale, covering the equivalent of the ontogenetic span of 16 years -8 months, consists of four segments each designed to test for the development of adaptive function during each of four sequential periods of four years. In ontogenesis the earlier developmental achievements are generally considered more fundamental for future adaptation, and are given more weight than later ones. In cases of adult patients the recovery process seems to depend equally on the reacquisition of the fundamental adaptive functions of each period, consequently the chronological equivalents in the growth of adaptive function are weighted equally through all parts of the scale. The scale emphasises cognitive or intellectual function but takes into account social and motivational components of development as well.

SEGMENT 1: $0-4$ YEARS

This segment consists of 97 items adapted from various infant scales, such as the Bayley (1969) Scales of Infant Development, the Boyd (1974) Developmental Progress Scale, and the Vineland Social Maturity Scale (Doll, 1953). About 1500 items from various infant scales were placed on 
cards. Two neurosurgeons and a psychologist rated these items as appropriate or inappropriate in assessing recovery. The 97 items that were rated as appropriate by all three raters were selected for the scale. These items were arranged in the order in which they appear chronologically in the infant scales. They appear to represent an adequate sample of the adaptive functions acquired in the first four years of life and to represent recovery through the end of the period of posttraumatic amnesia.

The examiner starts the examination with an item that appears to be within the patient's capacity, then continues with the easier items until the patient shows four successive passes. The examination proceeds with the more advanced items until there are four successive failures. It can be assumed that the items below the four successes and above four failures would all be passed or failed respectively.

SEGMENT 2: 4-8 YEARS

This segment consists of 20 items selected from the Stanford-Binet Intelligence Scale (Terman and Merrill, 1973) from age levels 4-9 years inclusive. The Binet test, even as originally conceived, was designed to determine readiness for school learning. Our selection of the Stanford-Binet items from this age period was guided by the widely accepted assumption that they represent the basic conceptual requirements for achieving the more complex adaptive skills. The emphasis in these items is on cognitive skills basic to adaptive function. The items test for the following abilities: making distinctions (noting differences); abstracting (noting similarities); following instructions; organising simple perceptual arrays.

We have chosen items from the Stanford-Binet rather than from other intelligence scales because, as Lezak (1976) points out, "individual Binet subtests fit well into larger test batteries constructed for specific research purposes and for the individual patient." Furthermore, we considered it appropriate to assess these particular cognitive functions in studying the recovery of patients with closed head injuries since they represent the kinds of functions not directly associated with localised cortical sites but those that seem sensitive to generalised brain injury (Yacorzinski, 1965).

SEGMENT 3: 8-12 YEARS

The three separate tests for this segment were selected because they show developmental improvement in basic information processing skills, and because they provided the opportunity to test whether or not the recovery process is differentiated with respect to type of injury.
Trail Making Test

The first part of the test requires the patient to draw lines in sequence from numbers 1 to 25 arranged randomly over an $8 \frac{1}{2}$ by 11 inch page. The second part of the test requires the patient to draw lines in alternating series consisting of 13 numbers and 12 letters. Score is the time to complete each part.

This test is taken from the Manual for the US Army Individual Test and has been used by Reitan (1958) as a test for the determination and localisation of brain damage. When used as a diagnostic measure in this way it produces too many false negative and false positive identifications (14 and $17 \%$ respectively). However, when we analyse what is involved in the performance of this task and adopt a developmental view, the Trail Making Test can provide some interesting and useful information about the patient's recovery.

In performing any task requiring the serial organisation of behaviour, each of the patient'so responses has to be determined by the use of information derived from the previous responses.? Warrington (1970) cites a study by Stevenson which showed that patients with unilateral frontalo lobe lesions performed significantly more poorlyo on sequentially ordered tasks than those with $\stackrel{\complement}{\mathbb{\perp}}$ lesions in other regions of the cortex. Right ando $z$ left frontal lobe patients were similarly impaired.

We have developed age norms for the Trai Making Test on a sample of 174 subjects ranging in age from 7 to 17 years. These norms are available on request. The test can thus be used to make a developmental assessment of recovery. Also by comparing the recovery on this task with that of the other two tests used in this segment it becomes possible to determine whether recovery is general or global or whether it is differentiated as to specific aspects of function.

\section{Road Map Test of Direction Sense}

The ability to differentiate right and left progresses through a developmental sequence (Swanson and Benton, 1955). First appears the ability to make the right-left (R-L) discrimination of one's own body (complete by about age 7 years). The R-L discrimination on a person facing the subject does not develop until about age 12 years. The Standardised Road Map Test of Direction Sense (Money, 1965) evaluates the more complex skill in R-L orientation-namely, "that of orienting oneself toward right and left simultaneously with coming and going in space and then projecting this orientation onto a flat surface, as in following a route on a map"(p. 16). The test requires the 
subject to identify the direction of $32 \mathrm{R}-\mathrm{L}$ turns on a street map.

Benton (1959) asserts that the ability of right-left orientation is dependent on language processes. The development of this orientation skill seems to be related to the process of cerebral hemispheric lateralisation.

We have included the Standardised Road Map Test of Direction Sense in our test battery because it seems to be a reflection of the gradual development of hemispheric specialisation. By using the age norms provided in the manual, one is able to obtain a measure of the recovery of the abstract referencing function of language symbols.

\section{Stroop Color-Word Test}

Although there are many forms of this test measuring the effects of perceptual interference, we have adopted the form proposed by Comalli et al. (1962) because they have provided age norms. We have supplemented their norms by testing a sample of 174 subjects ranging in age from 7 to 17 years. Our results are remarkably close to those obtained by Comalli et al. Age norms for this form of the Color-Word test are available on request.

The test consists of three cards $(24 \times 24 \mathrm{~cm})$. Card 1 consists of 100 colour words (red, blue, and green) which the subject reads. Card 2 has 100 small colour rectangles (red, blue, and green) which the subject names. Card 3 consists of 100 colour words but these words are printed in ink, the actual colour of which conflicts with that designated by the printed word. Thus, the word red is printed in blue or green ink but not in red ink. The subject must name the colour of the ink and ignore the printed colour word. Score is the amount of time for each card.

In order to perform well on card 3 the subject is required to inhibit word reading and to keep it subordinate to the more deliberate process of colour naming. This task represents the kind of complex adjustment necessary in paying selective attention to the environment and in maintaining an inhibitory state with respect to one response tendency-namely, reading-while at the same time proceeding actively in making a contradictory response-namely, colour naming. Developmentally, this skill, which involves selective inhibition and activation, seems to depend on the general state of superordination of higher cortical centres over the subcortical centres. The test thus seems appropriate to measure the reacquisition of this superordination in the recovery from serious head injury.

The three tests in this segment, Trail Making, Direction Sense, and Stroop Color-Word Test, can be used to determine whether the pattern of recovery is generalised or whether different types of injury lead to differentiated patterns of recovery for different functions.

SEGMENT 4: FINAL STAGES OF MATURE ADAPTIVE FUNCTION

For this segment we have selected a test that uses various concepts, skills, and information processing in a co-ordinated way to carry out everyday activities. This test is the REAL (Reading/Everyday Activities in Life) developed by Lichtman (1972). The test consists of nine subparts, such as identifying road signs by their function, reading a television programme matrix, reading the want-ad section of a newspaper, and so on. The test provides norms that differentiate the functionally illiterate, marginal, and functionally literate. Instructions are given on a cassette tape, and after initial instruction the test is self-administered and self-paced.

Further details on the several tests of the battery used for the Developmental Assessment of Recovery from Serious Head Injury (DARSHI), and instructions for administering and scoring, are available on request.

\section{Application of assessment procedure}

A number of patients with closed head injury have been evaluated sequentially using the various segments of our assessment procedure. We have found that the procedure is practical and appears to be valid. A few patients have been examined seven or eight times during the first 60 to 90 days of recovery and once or twice several months after discharge. A summary of one such patient's recovery is presented.

A 20 year old man (MP) sustained a head injury in a car accident. On admission, neurological examination showed that there was no response to verbal command but there was appropriate withdrawal of all extremities to painful stimuli. In addition, there was a mild but definite left hemiparesis including the face. The right pupil was larger than the left but both responded to light. Oculocephalic reflexes were intact; these was a tendency for adversive gaze to the right. After admission to hospital he underwent elevation and debridement of his skull fracture. At the time of surgery cerebral contusion was noted. The day after admission, mild left sided decerebrate posturing was noted, and the patient underwent a repeat CAT scan which showed diffuse swelling with no evidence of haematoma. Six days after injury he moved his right extremities appropriately to verbal 
command, but a marked left hemiparesis, arm greater than leg, persisted. Eight days after injury he was following command but marked left hemiparesis persisted. Ten days after injury he was responding well to command, and improvement of his left hemiparesis was seen. Seventeen days after injury the patient was able to say single words, and was noted to be more alert. In addition, slow but definite improvement of his left hemiparesis was noted. Thirty-seven days after injury there was steady improvement in his level of consciousness, speech, and left hemiparesis. The patient was discharged 67 days after injury. He was noted to be mildly ataxic but he was able to walk without assistance.

On the first neuropsychological assessment, 17 days after injury, the patient's level of function was at the 12 month equivalent. Ten days later his performance improved to the 4 year level. On day 27 after injury he was started on programmes of physical, occupational, and speech therapy, and at day 39 his performance on the Binet segment of the test battery placed him at an age equivalent of 94 months. One day later he was tested on two of the three tests of the third segment of the battery. On the Trail Making test his score was equivalent to the 8 year level, and on the test of directional sense his performance was at the 13

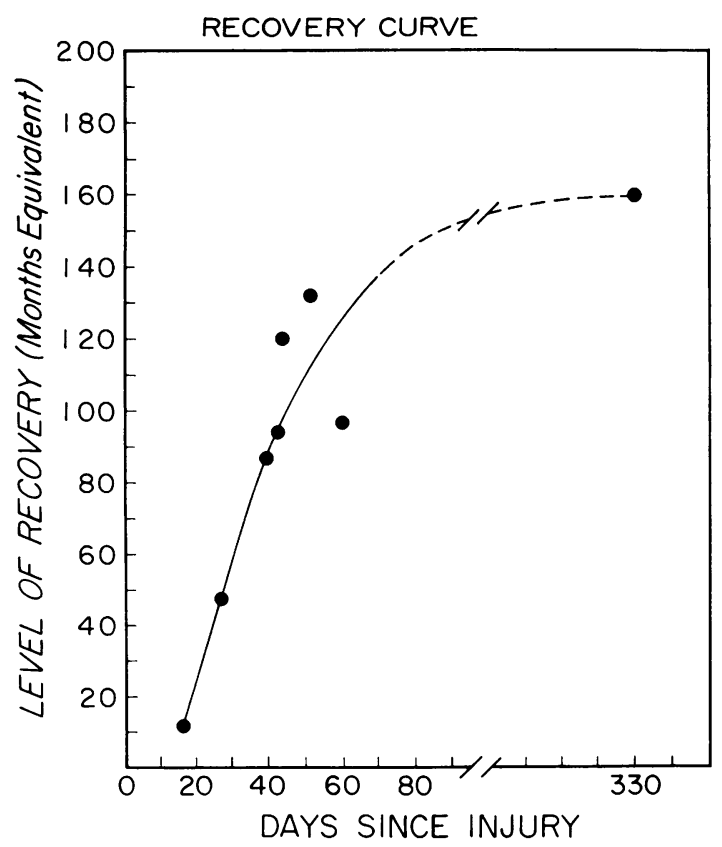

Figure Level of recovery in relation to days since injury in patient $M P$. year level. He was assigned a combined score of 10 years. His Color-Word scores at 50 and 52 days were at the 7 and 9 year levels respectively, while his score on the Directional Sense Test remained at age 13 years. At 60 days, a few days before discharge, his scores on all three tests had regressed somewhat, 7 years on Trail Making and the Color-Word test, and 8 years on the test of Directional Sense. On this last test he seemed to have become confused half way through and could not reorient himself.

The patient was seen 11 months after his injury, and was tested on the three tests of segment three. His scores ranged from 9 years on the Stroop interference card to 15 years on the test of Directional Sense. His overall performance was at the 13 year level. In addition, he showed some immature personality characteristics such as uncontrolled sporadic giggling. Although his premorbid functioning was judged to be marginal, we estimated it to be equivalent to the level we have termed basic adaptive function. Thus his recovery was still considerably short of that level. The course of this patient's recovery from 17 days to 11 months after injury is shown in the Figure.

\section{Discussion}

The changes in function as revealed by the sequential assessment by our procedure suggest that the course of recovery generally parallels ontogeny. The early stages are marked by rapid improvement. This is followed by a period of decelerating rate of improvement until the asymptote is reached. Newcombe et al. (1975) described a similar course of recovery in several dyslexic patients, and Knill-Jones (1975) suggests that the idealised recovery curve should follow this same pattern. However, ontogeny and recovery differ in two particular respects. First, patients seem to recover context-free responses before they become able to apply these in context-dependent situations. Thus, patients can recite the days of the week in sequence and tell what day comes before or after a specified day before they demonstrate a firm orientation to the current flow of time; they can identify right and left before they can consistently put their shoes on corresponding feet. Second, the functions necessary for performance on the latter half of segment 2 (items from the Stanford-Binet, testing for 7 to 8 year equivalence) seem to appear earlier than those necessary for performance at the same age level on the three tests in segment 3 -namely, Trail Making, Direction Sense, and Color-Word. Patients could complete all the items in segment 2 successfully, achieving an age 
equivalent of 8 years, while at the same time they could not perform at the 7 year level on the three tests of segment 3 . This finding is consistent with that reported by Gronwall and Sampson (1974) on a group of concussed patients. They found that concussion after head injury was associated with lowered performance in information processing tasks. It is also consistent with the findings of Mandleberg and Brooks (1975) that there is marked discrepancy between the verbal and performance subtcsts of the Wechsler Adult Intelligence Scale (19 IQ points in favour of the verbal). Our Binet items resemble the Wechsler verbal subtests, and our information processing tests resemble the performance subtests.

Some minor adjustments in the ordering of items within segments and in the co-ordination of segments 2 and 3 may be desirable ultimately in order to make the scale conform more precisely to the recovery process observed. However, our findings thus far indicate that the developmental parallel is generally valid in describing the neuropsychological recovery process.

The performance of the patients on the three tests of segment 3 shows that the pattern of recovery is differentiated. Improvement in performance on the three tests was as follows: the ability to perform on Direction Sense recovers most rapidly, the ability to perform on the Trail Making test is slowest in recovery. This suggests that tasks requiring sequential ordering and visual scanning are most impaired in closed head injury while the abstract referencing function of language symbols and hemispheric specialisation is least impaired. Functions related to hemispheric specialisation are earlier ontogenetically than those demanding serial organisation. This suggests that the recovery pattern follows the ontogenetic sequence.

The recovery of the patients we have examined by means of the developmental assessment show marked individual differences both as to rate and final outcome. The findings indicate, however, that in general the course of recovery of adaptive function is relatively rapid at its onset, after regaining consciousness, and then appears to slow down markedly to reach an asymptote. Our findings suggest that the assertion of Jennett et al. (1976) that most of the recovery occurs in the first six months needs to be qualified. The study of the individual course of recovery by means of developmental assessment suggests that in some cases maximum recovery is achieved before six months, and in other cases recovery of adaptive function continues beyond that point.

The developmental approach to the assessment of recovery of adaptive function after serious head injury presented above permits objective assessment of recovery in early stages of post-traumatic amnesia, and unifies assessment during the early stages of recovery with assessments made during subsequent periods of recovery. This approach permits the analysis of neuropsychological recovery as a continual process. This analysis of recovery provides more precise indices for predicting eventual outcome. Developmental assessment makes it possible to correlate neurological and systemic clinical factors with neuropsychological measures during the course of recovery. A developmental approach to the study of recovery provides a more exact description of eventual outcomes than has been possible previously. For example, the Glasgow Coma Scale as developed by Jennett et al. (1976) classifies functional outcome into five broad categories: death, persistent vegetative state, severe disability, moderate disability, and good recovery. These categories do not permit immediate grasp of the qualitative functional level of survivors within each designation. A developmental test battery, such as presented here, permits a more precise description of outcome on a continuum, and allows for the determination of the relationship between patterns of recovery and the duration of improvement in adaptive function. Developmental descriptions can be useful in research investigations making comparative evaluations of different courses of treatment and rehabilitation both in short-term and long-term effects. Such developmental assessments may also have practical value in legal determinations of damage and in vocational planning.

This project was supported by Grant No NS 13042 from the National Institute of Neurological Communicative Diseases and Stroke, National Institutes of Health. We express our gratitude to the following individuals who contributed to the investigation: Dr R. Ghiulamila, Dr A. J. Popp, P. Donahue, A. Van Deusen, D. Van Deusen, $M$. Booth, and Professor R. Pruzek.

\section{References}

Bayley, N. (1969). Manual of the Bayley Scales of Infant Development. The Psychological Corporation: New York.

Benton, A. L. (1959). Right-Left Discrimination and Finger Localisation. Hoeber-Harper: New York.

Bond, M. R. (1975). Assessment of the psychosocial outcome after severe head injury. In Outcome of Severe Damage to the Central Nervous System. Edited by R. Porter and D. W. Fitzsimons. Ciba Foundation Symposium 34 (new series). Elsevier: Amsterdam. 
Bond, M. R., and Brooks, D. N. (1976). Understanding the process of recovery as a basis for the investigation of rehabilitation for the brain injured. Scandinavian Journal of Rehabilitation Medicine, 8 , 127-133.

Boyd, R. D. (1974). The Boyd Developmental Process Scale. Inland Countries Regional Center: San Bernardino, California.

Brooks, D. N. (1974a). Recognition memory and head injury. Journal of Neurology, Neurosurgery, and Psychiatry, 37, 794-801.

Brooks, D. N. (1974b). Recognition memory after head injury: a signal detection analysis. Cortex, 10, 224-230.

Brooks, D. N. (1975). Long and short term memory in head injured patients. Cortex, 11, 329-340.

Brooks, D. N. (1976). Wechsler Memory Scale performance and its relationship to brain damage after severe closed head injury. Journal of Neurology, Neurosurgery, and Psychiatry, 39, 593-601.

Christenson, A. L. (1975). Luria's Neuropsychological Investigation: Text, Manual, and Test Cards. Spectrum (Halsted Press): New York.

Comalli, P. E., Wapner, S., and Werner, H. (1962). Interference effects of Stroop Color-Word test in childhood, adulthood and aging. Journal of Genetic Psychology, 100, 47-53.

Doll, E. A. (1953). A Manual for the Vineland Social Maturity Scale: The Measurement of Social Competence. Educational Test Bureau: Minneapolis.

Graham, D. I., and Adams, J. H. (1971). Ischaemic brain damage in fatal head injuries. Lancet, 1 , 245-266.

Gronwall, D. M. A., and Sampson, H. (1974). The Psychological Effects of Concussion. Auckland University Press: Auckland, New Zealand.

Hannay, H. J., Levin, H. S., and Grossman, R. G. (1977). Closed head injury and recognition memory. Paper presented at European meeting of International Neuropsychological Society, Oxford, England.

Jennett, B., Teasdale, G., Braakman, R., Minderhoud, J., and Knill-Jones, R. (1976). Predicting outcome in individual patients after severe head injury. Lancet, 1, 1031-1034.

Knill-Jones, R. P. (1975). Mechanisms of recovery: general discussion. In Outcome of Severe Damage to the Central Nervous System. Edited by R. Porter and D. W. Fitzsimons. Ciba Foundation Symposium, 34 (new series). Elsevier: Amsterdam.

Levin, H. S., Grossman, R. G., and Kelly, P. J. (1976). Short term recognition memory in relation to severity of head injury. Cortex, 12, 175-182.

Lezak, M. D. (1976). Neuropsychological Assessment. Oxford University Press: New York.

Lichtman, M. (1972). Reading/Everyday Activities in Life: Examiner's Manual. CAL Press: New York.

Luria, A. R., and Majovski, L. V. (1977). Basic approaches used in American and Soviet clinical neuropsychology. American Psychologist, 32, 959-968.
Mandleberg, I. A., and Brooks, D. N. (1975). Cognitive recovery after severe head injury. I. Serial testing on the Wechsler Adult Intelligence Scale. Journal of Neurology, Neurosurgery, and Psychiatry, 38, 1121-1126.

Mandleberg, I. A. (1975). Cognitive recovery after severe head injury. II. Wechsler Adult Intelligence Scale during post-traumatic amnesia. Journal of Neurology, Neurosurgery, and Psychiatry, 38, 11271132.

Money, J. (1965). A Standardized Road-Map Test of Direction Sense. The Johns Hopkins Press: Baltimore.

Najenson, T., Mendelson, L., Schechter, I., David C., Mintz, N., and Grosswasser, Z. (1973). Rehabilitation after severe head injury. Scandinavian Journal of Rehabilitation Medicine, 5, 1-10.

Najenson, T., Mendelson, L., Schechter, I., David, C., Mintz, N., and Grosswasser, Z. (1974). Rehabilitation after severe head injury. Scandinavian Journal of Rehabilitation Medicine, 6, 5-14.

Najenson, T., Grosswasser, Z., Stern, M., Schechter, I., David, C., Berghaus, N., and Mendelson, L. (1975). Prognostic factors in rehabilitation after severe head injury. Scandinavian Journal of Rehabilitation Medicine, 7, 101-105.

Newcombe, F., Hiorns, R. W., Marshall, J. C., and Adams, C. B. T. (1975). Acquired dyslexia: patterns of deficit and recovery. In Outcome of Severe Damage to the Central Nervous System. Edited by R. Porter and D. W. Fitzsimons. Ciba Foundation Symposium 34 (new series). Elsevier: Amsterdam.

Reitan, R. M. (1958). Trail making test and brain damage. Perceptual and Motor Skills, 8, 271-276.

Reitan, R. M., and Davison, L. A. (1974). Clinical Neuropsychology: Current Status and Applications. Winston/Wiley: New York.

Ruesch, J., and Moore, B. E. (1943). Measurement of intellectual function in the acute stage of head injury. Archives of Neurology and Psychiatry (Chicago), 50, 165-170.

Russell, E. W., Neuringer, D. C., and Goldstein, G. (1970). Assessment of Brain Damage. A Neuropsychological Key Approach. Wiley-Interscience: New York.

Swanson, R., and Benton, A. L. (1955). Some aspects of the genetic development of right-left discrimination. Child Development, 26, 123-133.

Terman, L. M., and Merrill, M. A. (1973). StanfordBinet Intelligence Scale: Manual for the Third Revision Form L-M. Houghton-Mifflin: Boston.

Warrington, E. K. (1970). Neurological deficits. In The Psychological Assessment of Mental and Physical Handicaps. Edited by P. M. Mittler. Methuen: London.

Yacorzinski, C. R. (1965). Organic mental disorders. In Handbook of Clinical Psychology. Edited by B. B. Wolman. McGraw-Hill: New York. 